\section{Morbidity after adenotonsillectomy for paediatric obstruc- tive sleep apnoea syndrome: waking up to a pragmatic approach}

\section{J Laryngol Otol 2007;121:809-17}

Dear Sirs

The above review article on the management of paediatric obstructive sleep apnoea (OSA) ${ }^{1}$ is welcome, as it serves to raise awareness of a condition which can be associated with long term cardiorespiratory sequelae (e.g. pulmonary hypertension, cor pulmonale, dysrhythmias and intracranial hypertension). Of more immediate concern are the associated neuropsychological complications, such as deficits in attention span, executive function, visual attention, general conceptual ability and phonological processing (the latter being crucial for reading skills development). ${ }^{2}$ In addition, affected children and their families have a significantly reduced quality of life. ${ }^{3}$

The review concludes that children classified as high risk should be operated upon in a dedicated paediatric specialist centre with intensive care facilities, because of the increased chance of post-operative respiratory complications (in particular, pulmonary oedema as a result of removing the 'natural' form of positive end-expiratory pressure). Children at moderate to low risk may undergo surgery at their district general hospital, provided facilities for administering continuous positive airway pressure are available on site for those in the moderate risk group.

In the review, the authors proposed an OSA grading system whereby an apnoeic-hypopnoeic index (AHI) score of between five and 10 is considered as mild OSA, 10 to 20 as moderate, and more than 20 as severe. This is somewhat dissimilar to the American Academy of Sleep Medicine system of OSA grading, ${ }^{4}$ which states that an AHI of five to 15 is mild, 15 to 30 is moderate and more than 30 is severe.

However, the latter classification refers to adult OSA, which is distinctly different from childhood OSA. Whilst the authors were aware of the absence of an internationally accepted paediatric OSA classification, we feel compelled to draw readers' attention to the fact that, in children, even one apnoeic episode may lead to the diagnosis of OSA. That is one of the reasons why adult classifications of OSA on the basis of AHI cannot be extrapolated to children; in other words, as the Section on Pediatric Pulmonology, Subcommittee on OSA syndrome (OSAS) states, 'Normative standards for PSG [polysomnographic] determination of OSAS have been chosen on the basis of statistical distribution of data, and it has not been established that those standards have any validity as predictors of the occurrence of complications'.

Therefore, we feel that the proposed paediatric grading system in the review may need amending, perhaps in the light of the technical report by Schechter et al..$^{5}$ Finally, the fact that in the UK 'facilities for administering continuous positive airway pressure' are largely limited to paediatric intensive therapy units or high dependency units essentially undermines the value of having a 'moderate risk' OSA group in children for the purposes of surgical intervention.

\section{R Persaud \\ Z Awad* \\ C Georgalas $\dagger$}

From the Northwick Park Hospital, Harrow, UK, the *Basildon and Thurrock University Hospital, UK, and the ${ }^{\dagger}$ Academic Medical Center, Amsterdam, Netherlands.

\section{References}

1 Leong AC, Davis JP. Morbidity after adenotonsillectomy for paediatric obstructive sleep apnoea syndrome: waking up to a pragmatic approach. J Laryngol Otol 2007;121: $809-17$

2 O'Brien LM, Mervis CB, Holbrook CR, Bruner JL, Klaus CJ, Rutherford J et al. Neurobehavioral implications of habitual snoring in children. Pediatrics 2004;114:44-9

3 Georgalas C, Babar-Craig H, Arora A, Narula A. Health outcome measurements in children with sleep disordered breathing. Clin Otolaryngol 2007;32:268-74

4 Gay P, Weaver T, Loube D, Iber C. Evaluation of positive airway pressure treatment for sleep related breathing disorders in adults. Sleep 2006;29:381-401

5 Schechter MS. Technical report: diagnosis and management of childhood obstructive sleep apnea syndrome. Pediatrics 2002;109(4):e69

Author's reply

Dear Sirs I thank Persaud et al. for their letter regarding my and Davis's recent review on paediatric obstructive sleep apnoea syndrome (OSAS). ${ }^{1}$

The paediatric OSAS severity grading mentioned in our review was cited from a key reference by Guilleminault et al. ${ }^{2}$ and was suggested as a guide to assessment of a condition without universally accepted, consistent and reliable diagnostic criteria. This classification has also previously been used in an attempt to stratify those children who might require elective admission to the paediatric intensive care unit following adenotonsillectomy for OSAS. ${ }^{3}$ We agree that '.. normative standards for their polysomnographic determination have been chosen on the basis of statistical distribution of data'; whether those standards have any validity as predictors of the occurrence of complications currently remains unknown. This important point was emphasised at the end of our section discussing the usefulness of polysomnography in the diagnosis of paediatric OSAS, concluding that polysomnography appears to be most useful to confirm the diagnosis of OSAS, where history and examination fail to concur, and to document the severity of OSAS in certain high risk groups. Our suggested management algorithm was based on these key points and was in line with the recommendations made by the Section on Pediatric Pulmonology and Subcommittee on OSAS. ${ }^{4}$

Persaud et al. state that '... even one apnoeic episode may lead to the diagnosis of OSA'. However, it would be prudent to bear in mind that an episode of witnessed apnoea may not necessarily lead to associated desaturations ${ }^{5}$ further confounding the issue of what can be strictly considered as, and diagnosed as, obstructive sleep apnoea itself, rather than the less severe disorders of obstructive hypoventilation and upper airway resistance syndrome included within the whole spectrum of OSAS.

Persaud et al. cite Schechter's technical report which, upon careful reading, actually concludes its discussion on polysomnography as follows.

First published online 7 June 2007. 
Having stated these points, additional analysis of the validity of alternative diagnostic approaches will be done assuming polysomnography as the gold standard. One additional benefit of overnight polysomnography is that in addition to establishing the diagnosis of OSAS, polysomnography also may be used to determine its severity. It has been suggested that the severity of OSAS is an important predictor of complications, particularly in the immediate postoperative period. ${ }^{6}$

Once again, this concurs with our review.

We accept Persaud and colleagues' observation that most UK facilities for administering nasal continuous positive airway pressure (CPAP) would probably be found within the environs of a paediatric high dependency unit or intensive therapy unit. If CPAP is unavailable, we suggested in our algorithm that it might be safer to perform adenotonsillectomy procedures on children in the moderate risk group at an institution offering this option. However, local and regional resources as well as anaesthetic preferences would naturally dictate that there be a degree of variability and flexibility in the management of these patients.

A Leong

JP Davis
Department of Otolaryngology,

Medway Maritime Hospital,

Gillingham, Kent

\section{References}

1 Leong AC, Davis JP. Morbidity after adenotonsillectomy for paediatric obstructive sleep apnoea syndrome: waking up to a pragmatic approach. J Laryngol Otol 2007;121: 809-17

2 Guilleminault C, Black JE, Palombini L, Obayon M. A clinical investigation of obstructive sleep apnoea syndrome (OSAS) and upper airway resistance syndrome (UARS) patients. Sleep Med 2000;1:51-6

3 Walker P, Whitehead B, Rowley M. Criteria for elective admission to the paediatric intensive care unit following adenotonsillectomy for severe obstructive sleep apnoea. Anaesth Intensive Care 2004;32:43-6

4 Clinical practice guideline: diagnosis and management of childhood obstructive sleep apnea syndrome. Pediatrics 2002;109:704-12

5 Uliel S, Tauman R, Greenfeld M, Sivan Y. Normal polysomnographic respiratory values in children and adolescents. Chest 2004;125:872-8

6 Schechter MS. Technical report: diagnosis and management of childhood obstructive sleep apnea syndrome. Pediatrics 2002;109:e69 [8 June 2008] 